\title{
Targeting efficiency and biodistribution of biotinylated-EGF-conjugated gelatin nanoparticles administered via aerosol delivery in nude mice with lung cancer
}

\author{
Ching-Li Tseng, Steven Yueh-Hsiu Wu, Wen-Hsi Wang, Cheng-Liang Peng, Feng-Huei Lin*, \\ Chien-Cheng Lin, Tai-Horng Young, Ming-Jium Shieh
}

Institute of Biomedical Engineering, National Taiwan University, No. 1, Section 1, Ren-ai Road, Taipei 100, Taiwan, ROC

\section{A R T I C L E I N F O}

\section{Article history:}

Received 19 December 2007

Accepted 11 March 2008

Available online 23 April 2008

\section{Keywords:}

Nanoparticle

Gelatin

In vivo test

Lung cancer

Drug delivery

EGF

\begin{abstract}
A B S T R A C T
Lung cancer is the most malignant cancer today; in order to develop an effective drug delivery system for lung cancer therapy, gelatin nanoparticles (GPs) were modified with NeutrAvidin ${ }^{\text {FITC-biotinylated }}$ epidermal growth factor (EGF) to form EGF receptor (EGFR)-seeking nanoparticles (GP-Av-bEGF). Aerosol droplets of the GP-Av-bEGF were generated by using a nebulizer and were delivered to mice model of lung cancer via aerosol delivery.

Analysis of the aerosol size revealed that $99 \%$ of the nanoparticles after nebulization had a mass median aerodynamic diameter (MMAD) within the suitable range $(0.5-5 \mu \mathrm{m})$ for lower airway deposition. The safety of inhaled nanoparticles was examined by lung edema and myeloperoxidase (MPO) activity assay. There's no finding suggestive of acute lung inflammation following inhalation. The fluorescence images obtained from live mice showed that the GP-Av-bEGF could target the cancerous lungs in a more specific manner. Fluorescence analysis of the organs revealed that the GP-Av-bEGF was mainly distributed in cancerous lungs. In contrast, nanoparticle accumulation was lower in normal lungs. The histological results indicated that the fluorescent GP-Av-bEGF was colocalized with the anti-EGFR-immunostain due to EGFR binding.

The results of this study revealed that GP-Av-bEGF could target to the EGFR-overexpression cancer cells in vivo and may prove to be beneficial drug carriers when administered by simple aerosol delivery for the treatment of lung cancer.
\end{abstract}

(c) 2008 Elsevier Ltd. All rights reserved.

\section{Introduction}

Lung cancer is one of the most harmful forms of cancer. The longterm survival rate of lung cancer patients treated by conventional modalities such as surgical resection, radiation, and chemotherapy remains far from satisfactory. Systemic drug delivery is rarely successful because only a limited amount of the chemotherapeutic drug targets lung tumor sites, even when administered at a high dose [1]. Most of the chemotherapeutic drugs act on normal cells, inhibiting their growth; this makes the patient extremely weak and can even result in death. Therefore, accurate delivery of chemotherapeutic drugs to the tumor site is the most crucial step for increasing the survival rate of lung cancer patients. Intravenous (i.v.) injection of protein-based drugs and anticancer pharmaceuticals, with or without drug carriers, is the major delivery method employed for chemotherapy [2]. However, drugs encapsulated in nanocarrier were mostly accumulated in the reticuloendothelial system (RES) such as liver or spleen before they reach the intended target site [3].

\footnotetext{
* Corresponding author. Tel.: +886 2 23123456x1568; fax: +886223940049.

E-mail address: double@ntu.edu.tw (F.-H. Lin).
}

The direct delivery of chemotherapeutic agents to the lungs offers a novel therapeutic approach for lung cancer patients. By the aerosol method, chemotherapeutic drugs can be efficiently and noninvasively delivered to lung cancer area by inhalation [4]. Thus, the drug can directly exert its effects on lung cancer cells before it is degraded or metabolized. Targeted aerosol delivery can also increase the retention time of the drug in the lungs; this improves the pulmonary receptor occupancy at the expense of systemic exposure, thereby reducing the systemic side effects of the drug $[5,6]$. In addition, this method could eliminate patient complaints associated with the daily administration employed in i.v. injection.

Aerosol therapy employing a particulate drug carrier system is becoming a popular method for the delivery of therapeutic compounds. Although many materials have been used for constructing nanoparticles to serve as carriers for anticancer drugs, not all these materials have proved to be very promising when applied to the human body. Poly(lactic-co-glycolic acid) (PLGA) is a very common biodegradable polymer used for drug delivery. However, its biodegradation rate is extremely slow, and it is therefore unsuitable for pulmonary drug delivery, especially in cases where frequent dosing is required [7]. Leakage has always been a limitation of 
liposome nanoparticles prepared by nebulization as aerosols for inhalation therapy [8].

Gelatin is a biodegradable polymer that exhibits excellent biocompatibility, plasticity, and adhesiveness [9]. Its degradation rate can be regulated by the degree of cross-linking. The functional groups on gelatin nanoparticles (GPs), such as carboxyl, hydroxyl, and amino groups, are available for conjugation with ligands to bring about surface modifications. Gelatin microspheres have been used for the effective delivery of salmon calcitonin to the pulmonary system [10]. Several investigations have confirmed the aerodynamic stability of gelatin aerosol particles generated by using a nebulizer $[6,10]$. In the present study, GPs were generated as drug carriers (cisplatin) to be administered via inhalation for the treatment of lung cancer. The developed GPs were surface modified NeutrAvidin ${ }^{\text {FITC }}$-biotinylated epidermal growth factor (GP-Av-bEGF) to direct them to the tumor site, and they were dispersed in an aerosol; they were then delivered in vivo to lung cancer cells in nude mice via inhalation. Fluorescence imaging and histological observation were performed to analyze the biodistribution and targeting efficiency of the nanoparticles in vivo via aerosol delivery. The particle size of the nebulized nanoparticles was assessed by using a dust monitor. Scanning electron microscopy (SEM) was performed to examine the morphology of the nebulized GPs. An acute lung injury assay was performed to evaluate the safety of the developed nanoparticles. The lung wet-to-dry $(W / D)$ weight ratio was determined to examine lung edema, and the myeloperoxidase (MPO) activity assay was performed to assess neutrophil infiltration [11].

\section{Materials and methods}

\subsection{Reagent and chemicals}

Gelatin type A (derived from porcine skin, bloom 175), Ham's F12K medium, Bicinchoninic Acid (BCA) protein assay kit, bovine serum albumin (BSA) ethylenediaminetetraacetic acid (EDTA), hexadecyltrimethylammonium bromide (HETAB), hematoxylin and eosin were purchased from Sigma (Saint Louis, MO, USA). Fetal calf serum (FCS) was from Biological industries (Kibbutz Beit Haemek, Israel). Trypsin-EDTA, penicillin/streptomycin and phosphate-buffered saline (PBS) were obtained from Gibco/BRL (Gaithersburg, MD, USA). NeutrAvidin ${ }^{\mathrm{TM}}$ Fluorescein Conjugated (NeutrAvidin ${ }^{\text {FITC }}$ ), $m$-maleimidobenzoyl- $N$-hydroxysulfosuccinimide ester (Sulfo-MBS), Sulfo-NHS-LC-biotin, 2-iminothiolane- $\mathrm{HCl}$, D-Salt ${ }^{\mathrm{TM}}$ Dextran desalting columns and $\mathrm{EZ}^{\mathrm{TM}}$ Biotin Quantization Kit were purchased from Pierce (Rockford, IL, USA). Human recombinant epidermal growth factor (EGF) and mouse (monoclonal) anti-human EGF receptor (EGFR) antibody cocktail were obtained from BioSource (Camarillo, CA, USA). Amicon ${ }^{\circledR}$ ultra centrifugal filter device was from Millipore (Billerica, MA, USA). Super Sensitive Non-Biotin HRP Detection System was from BioGenex (San Ramon, CA, USA). Tissue-Tek ${ }^{\circledR}$ OCT compound was obtained from Sakura Finetek (Sakura, Tokyo, Japan). All other chemicals were from Sigma-Aldrich in reagent grade.

\subsection{Preparation of gelatin nanoparticles with conjugated biotinylated EGF}

\subsubsection{Preparation of gelatin nanoparticles (GPS)}

GPs were prepared by the desolvation method, which is briefly described as follows [12]. Five milliliters of $5 \%(w / v)$ aqueous gelatin solution was heated to $50{ }^{\circ} \mathrm{C}$ followed by the addition of $5 \mathrm{ml}$ of acetone. The resulting supernatant was discarded and the precipitate redissolved at $50{ }^{\circ} \mathrm{C}$. Twelve milliliters of acetone was then added to the redissolved gelatin solution at $\mathrm{pH} 2.5$. The nanoparticles were crosslinked by the addition of glutaraldehyde $(0.04 \%)$ and stirred at $1000 \mathrm{rpm}$ overnight. Finally, acetone was removed by vacuum drying, and the fabricated GPs were purified and resuspended in deionized water. These particles were stored at $4{ }^{\circ} \mathrm{C}$ for further applications.

\subsubsection{NeutrAvidin ${ }^{\text {FITC }}$ conjugation to the surface of GPS}

Two steps were required in order to complete the conjugation process. Initially, amino groups borne on the surface of GPs were converted to thiol groups by the addition of 2-iminothiolane. These thiol groups were subsequently used as functional groups for ligand conjugation. The GPs in deionized water were initially placed in a dialysis membrane bag and dialyzed against sodium phosphate buffer containing 10 mм EDTA ( $\mathrm{pH}$ 8.0). Following dialysis, $1 \mathrm{ml}$ of gelatin nanoparticle solution $(8 \mathrm{mg} / \mathrm{ml})$ was reacted with 2 -iminothiolane $(28 \mathrm{~mm})$ for $1 \mathrm{~h}$ at $37^{\circ} \mathrm{C}$. The nanoparticles were washed three times with sodium phosphate buffer containing $10 \mathrm{~mm}$ EDTA and collected in Amicon Ultra-4 filter devices ( $M_{\mathrm{w}}$ cutoff, 30,000). As a result of this process, the thiol groups were introduced into the GPs. The introduced thiol groups were determined spectrophotometrically using the 5,5'-dithio-bis-(2nitrobenzoic acid) (DTNB) method [13]. Separately, NeutrAvidin ${ }^{\text {FITC }}$ was dissolved in sodium phosphate buffer ( $\mathrm{pH} 7.2$ ) containing $2 \mathrm{mg} / \mathrm{ml}$ of Sulfo-MBS for activation. The solution was mixed thoroughly and left to react at room temperature for $1 \mathrm{~h} \mathrm{[14]}$. The activated NeutrAvidin ${ }^{\text {FITC }}$ was purified on a gel filtration column. The activated NeutrAvidin ${ }^{\text {FITC }}$ solution was then mixed with the thiolated nanoparticles and allowed to react overnight at $4{ }^{\circ} \mathrm{C}$. The unbound NeutrAvidin ${ }^{\mathrm{FITC}}$ derivative was

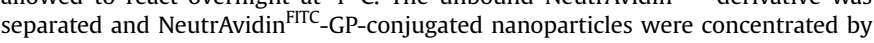
centrifugation using a centrifugal filter device $\left(M_{\mathrm{W}}\right.$ cutoff, 100,000). The complex of NeutrAvidin ${ }^{\text {FITC }}$-gelatin nanoparticles is hereafter abbreviated as GP-Av.

\subsubsection{Biotinylated EGF binding to NeutrAvidin ${ }^{\text {FITC }}$-GPS}

The biotinylation of EGF was performed prior to the conjugation with GP-Av, which is briefly described as follows. EGF was initially dissolved in phosphatebuffered saline (PBS, pH 7.0) and then added to a biotinylation reagent (Sulfo-NHSLC-biotin). The molar ratio of Sulfo-NHS-LC-biotin to EGF was 15:1 [14]. The solution was mixed and left to react at room temperature for $30 \mathrm{~min}$. Biotinylated EGF was separated by size exclusion chromatography through a D-salt dextran desalting column. The biotinylated EGF-containing eluate fractions were pooled, and the protein concentration of biotinylated EGF was measured using a BCA protein assay kit. The mole ratio of biotin binding with EGF was determined using an $\mathrm{EZ}^{\mathrm{TM}}$ Biotin Quantitation kit. Biotinylated EGF is hereafter abbreviated as bEGF. Following the

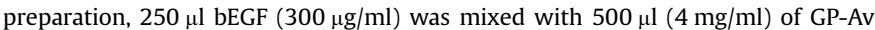
nanoparticles and incubated at $4{ }^{\circ} \mathrm{C}$ for $2 \mathrm{~h}$. The bEGF-conjugated GPs were washed 3 times with PBS and collected by centrifugation. The final nanoparticles are referred to as GP-Av-bEGF

\subsection{Cell and pulmonary tumor metastases}

Male nude mice, aged 5-6 weeks and weighing above $20 \mathrm{~g}$, were maintained under specific pathogen-free conditions and supplied with autoclaved mouse chow and water. The mice, housing facilities, feed, and animal use protocol were approved by the Institutional Animal Care and Use Committee of the National Taiwan University College of Medicine. A549 human lung adenocarcinoma cells were cultured in T-75 flasks using Ham's F12K medium supplemented with $10 \%$ fetal calf serum (FCS), $1 \%$ penicillin/streptomycin, and $1.5 \mathrm{~g} / \mathrm{l}$ sodium bicarbonate. All cultures were incubated in $5 \% \mathrm{CO}_{2}$ at $37{ }^{\circ} \mathrm{C}$. Having obtained a sufficient cell number, the A549 cells $\left(6 \times 10^{6}\right.$ cells suspended in $0.15 \mathrm{ml}$ PBS $)$ were injected into the tail vein of male BALB/CAnN.Cg-Foxn $1^{\mathrm{nu}} / \mathrm{CrlNarl}$ nude mice, in order to induce tumor in pulmonary tissue.

\subsection{Characterization of aerosol particles}

The size distribution of nebulized GPs, GP-Av, and GP-Av-bEGF, each at a concentration of $100 \mu \mathrm{g} / \mathrm{ml}$, was analyzed using a DUST monitor (DUST-check portable dust monitor, model 1.108; Grimm Labortechnik Ltd., Germany). The morphology of the developed nanoparticles was examined using a scanning electron microscope (SEM, S-2400; Hitachi, Japan). The developed nanoparticles were nebulized to an aerosol using a nebulizer (AP-100100; APEX, Taiwan). The original nanoparticle solution was dropped on the cover slide for the observation of nanoparticles. Aerosol droplets containing the developed nanoparticles were collected from the outlet of the nebulizer and dried on a cover slide. These cover slides were then coated with a gold film by sputtering physical vapor deposition (PVD) for SEM examination.

\subsection{In vivo aerosol delivery of GP-Av-bEGF}

The targeting efficiency of the developed GP-Av-bEGF in vivo was evaluated by an in vivo imaging system (IVIS ${ }^{\circledR}$ Imaging System 50; Xenogen, USA). Tumor-induced mice were divided into 3 groups and treated with PBS, GP-Av, or GP-Av-bEGF. The nanoparticle solution was nebulized to an aerosol and delivered to nude mice through inhalation. The mice were exposed to the aerosol in a sealed plastic box [1]. Five milliliters of nanoparticle solution in the reservoir of the nebulizer was consumed within $30 \mathrm{~min}$. The total deposited dose of GP-Av-bEGF was $5 \mathrm{mg} / \mathrm{kg} / \mathrm{mouse}$ for each exposure. Whole mouse images were obtained $1 \mathrm{~d}$ after aerosol delivery. The distribution of the developed GP-Av-bEGF in each organ was examined using the same imaging system. Normal mice and tumor-induced mice were all treated with GP-Av-bEGF nanoparticles $(n=4)$ using the same delivery process as described in the previous section. The mice were sacrificed at different time points $(0.5$ and $24 \mathrm{~h}$ ). Lungs, heart, liver, kidneys, spleen, and brain were harvested and cleaned using PBS. The relative intensity of fluorescence in each organ was equivalent to the concentration of fluorescent nanoparticles.

\subsection{Histological examination}

The tissues were harvested from the sacrificed mice and placed in tissue wells filled with Tissue-Tek OCT. The tissue wells were rapidly frozen on dry ice before being stored at $-70^{\circ} \mathrm{C}$. The frozen specimens were sectioned using a cryostat microtome (CM 3050S; Leica, Germany). The sectioned specimens were directly examined under a fluorescent microscope (Axiovert 100TV; Zeiss, Germany) in order to detect the location of the GP-Av-bEGF. Hematoxylin and eosin (H\&E)-stained specimens were 
used to check alveolar structure and tumor nodules. An anti-EGFR immunohistochemistry assay was used in order to label the EGFR-expressed cells. The cryosections were post-fixed with acetone for $10 \mathrm{~min}$ at $-20^{\circ} \mathrm{C}$ and washed with PBS. Nonspecific binding sites were blocked with $2 \%(\mathrm{w} / \mathrm{v})$ BSA solution in PBS prior to labeling with antibody. The specimens were washed several times with PBS, incubated overnight at $4{ }^{\circ} \mathrm{C}$ with a 1:200 dilution of monoclonal anti-EGFR primary antibody. The specimens were then stained using a commercial Super Sensitive Non-Biotin HRP Detection System (BioGenex, USA). All sections were counterstained with hematoxylin and mounted on a cover slide for optical microscopic examination.

\subsection{Acute lung injury assay}

\subsubsection{Wet to dry lung weight ratio}

The wet to dry lung weight ratio was used to check lung edema after nanoparticle treatment. One day after the mice had been treated by GP-Av-bEGF inhalation, the animals were euthanized by an intraperitoneal injection of overdose sodium pentobarbital. The right lung was harvested and weighed immediately. The dry weight of the right lung was subsequently determined by drying the tissue in a laboratory oven at $60^{\circ} \mathrm{C}$ for $2 \mathrm{~d}$. The wet/dry lung weight ratio was calculated in order to determine if nanoparticle treatment would cause lung edema.

\subsubsection{Myeloperoxidase assay}

Tissue myeloperoxidase (MPO) activity was used to quantitate neutrophil accumulation in the lung parenchyma of each experimental group. The left lung in $50 \mathrm{~mm}$ potassium phosphate buffer ( $\mathrm{PPB}, \mathrm{pH}$ 6.0) was chopped into pieces using a homogenizer. The homogenate was freeze-thawed 3 times and sonicated for $15 \mathrm{~s}$ on ice in order to further break down the pieces. After thawing, the sample was centrifuged for $10 \mathrm{~min}$ at $10,000 \times \mathrm{g}$ and $4{ }^{\circ} \mathrm{C}$. The resulting pellets were resuspended in $1 \mathrm{ml}$ HETAB solution ( $0.5 \%$ hexadecyltrimethylammonium bromide/PPB buffer). The MPO assay reagent comprised $0.05 \% \mathrm{H}_{2} \mathrm{O}_{2}$ and $0.19 \mathrm{mg} / \mathrm{ml} O$-dianisidine dihydrochloride in PPB, pH 6.0. A 10- $\mu$ l aliquot of the resuspended pellets and $290 \mu \mathrm{l}$ MPO assay reagent were mixed and allowed to react for $1 \mathrm{~min}$, after which absorbance was measured at $460 \mathrm{~nm}$ using an ELISA reader (SpectraMax M2; Molecular Devices, USA). The MPO activity was expressed by the following formula: MPO activity $=($ MPO value recovered from nanoparticle-treated mice $) /($ MPO value recovered from control mice without treatment) $\times 100(\%)$.

\subsection{Statistical analysis}

All data are presented as mean values $( \pm$ SEM). Statistical differences between groups were assessed using a one-way analysis of variance (ANOVA). The statistical significance for all tests was defined as $p \leq 0.05$.

\section{Results}

\subsection{Characterization of the nebulized GPS}

Schematic representation of the GPs with different degrees of modification is represented in Fig. 1(a). The particle sizes of the GPs, GP-Av, and GP-Av-bEGF measured by using a Photo-Correlated Spectrophotometer were all determined to be approximately $220 \mathrm{~nm}$ in previous report [15]. Following nebulization, the GPs, GP-Av, and GP-Av-bEGF were converted into aerosol particles. The size distribution of these aerosol particles was evaluated by using a dust monitor. As shown in Fig. 1(b), the sizes of the nebulized GPs, GP-Av, and GP-Av-bEGF (aerosol particles) all exhibited an extremely narrow distribution. More than $99 \%$ of all three GPs types were in the range of $0.5-5 \mu \mathrm{m}$ in size; this represents the optimal size range for delivery via inhalation to prevent deposition of the particles in the air track or their escape through the upper respiratory track.

Fig. 2(a) shows the morphology of the GPs, as determined by SEM. The GPs size was extremely uniform in the range 100-300 nm. Consistent with previous studies, the average size was approximately $200 \mathrm{~nm}$. The aerosol particles generated using the other two GPs types tested (GP-Av and GP-Av-bEGF) were morphologically identical to those generated using unmodified GPs when examined

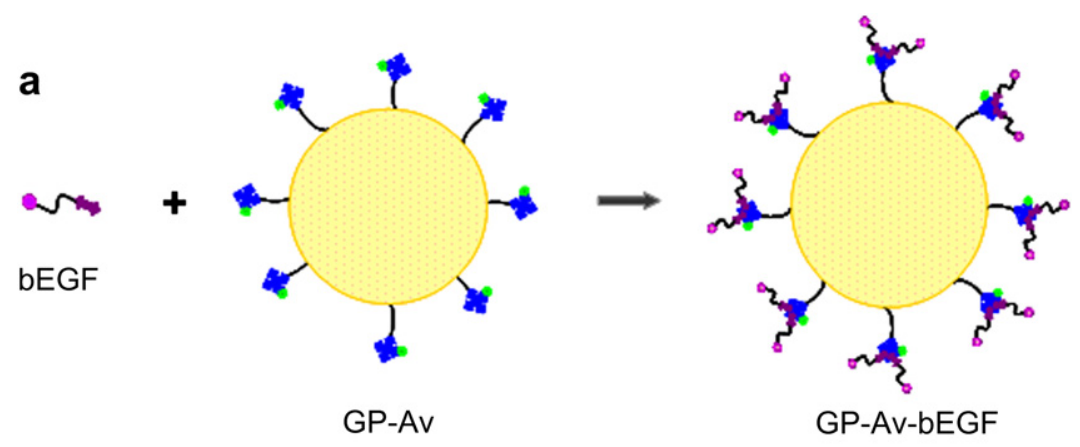

GPs: Gelatin nanoparticles

GP-Av: GPs surface modified with NeutrAvidin FITC

GP-Av-bEGF:GP-Av conjugated with biotinylated EGF

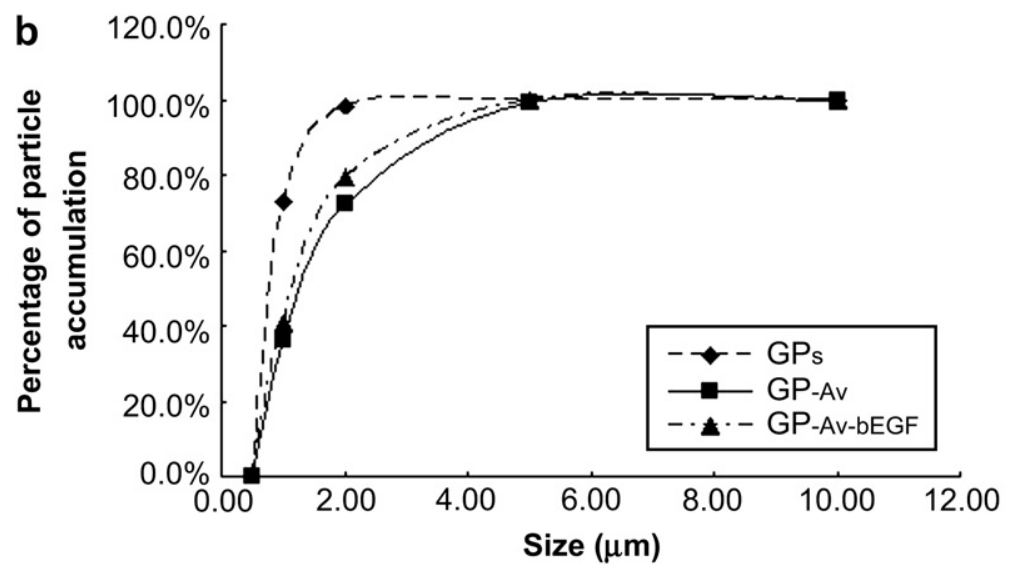

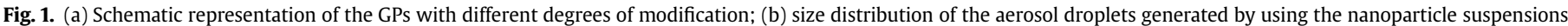
following nebulization. 
by SEM (data not shown). The results of the SEM examination were similar to those obtained by using a photo-related spectrophotometer. Following nebulization, the aerosol particles generated using the GPs were approximately $0.4-2 \mu \mathrm{m}$ in size (Fig. 2(b) red circles). As shown in Fig. 2(c), we also observed that after nebulization, some particles were not present in aggregates but as single nanoparticles, possibly due to their liberation from the aggregated clusters.

\subsection{Safety of aerosol nanoparticles}

To determine the safety of the developed nanoparticles, lung edema and inflammatory responses were checked based on the lung $W / D$ weight ratio and by performing the MPO assay, respectively. The lung $W / D$ weight ratio was $4.56 \pm 0.47$ in the normal mice treated with PBS (Table 1 ) and $4.17 \pm 0.24$ and $4.37 \pm 0.10$ in the normal mice treated with GP-Av-bEGF and the tumor-induced mice treated with GP-Av-bEGF, respectively. The three test groups did not differ significantly in the lung $W / D$ weight ratio $(p \leq 0.05)$. There should be no lung edema after GP-Av-bEGF delivery. An MPO activity assay was performed to detect the neutrophil accumulation in the lungs. The MPO activity in the normal mice treated with PBS was $99.98 \pm 7.41$. In the other two groups (normal mice treated with GP-Av-bEGF and tumor-induced mice treated with GP-AvbEGF), the MPO activity was $100.24 \pm 3.56$ and $96.82 \pm 2.37$, respectively (Table 1 ). No statistically significant difference was observed among the three groups in the MPO activity $(p \leq 0.05)$.

\subsection{In vivo targeting}

\subsubsection{Distribution of the nanoparticles in live mice}

Fig. 3 shows the distribution of the nanoparticles in live mice $24 \mathrm{~h}$ later following aerosol delivery. Tumor-induced mice under gas anesthesia were used to monitor the particle distribution in real time. The image in Fig. 3(a) corresponds to a PBS-treated mouse; almost no fluorescence was detected. The number of fluorescent spots obtained was greater for the mouse treated with GP-Av (Fig. 3(b)) than that for those treated with PBS. The mouse treated with GP-Av-bEGF yielded the strongest fluorescence signals among the three test groups. The nanoparticles largely accumulated in the tumor region of the lungs (Fig. 3(c)). This real-time imaging system may not accurately reveal the distribution of nanoparticles
Table 1

Results of acute lung injury assay

\begin{tabular}{lccc}
\hline Acute lung injury & $\begin{array}{l}\text { Normal group } \\
\text { PBS }\end{array}$ & $\begin{array}{l}\text { Normal group } \\
\text { GP-Av-bEGF }\end{array}$ & $\begin{array}{l}\text { Tumor-induced } \\
\text { group GP-Av-bEGF }\end{array}$ \\
\hline $\begin{array}{l}\text { Lung edema } \\
\text { (wet/dry ratio) }\end{array}$ & $4.56 \pm 0.47$ & $4.17 \pm 0.24$ & $4.37 \pm 0.10$ \\
MPO activity (\%) & $99.98 \pm 7.41$ & $100.24 \pm 3.56$ & $96.82 \pm 2.37$ \\
\hline
\end{tabular}

One-way ANOVA at the 0.05 level; the difference in the population means is significantly different from test difference.

especially in the deeper area of the body. Therefore, the next step was to measure the fluorescence intensity in the organs in order to further elucidate the path of the nanoparticles.

\subsubsection{Accumulation of the nanoparticles in organs}

The distribution profile of the GP-Av-bEGF in organs following aerosol administration in the mice is shown in Fig. 4. It is evident from Fig. 4(a) and (b) that the accumulation of fluorescent signals differed significantly between the normal and cancerous lungs at 0.5 and $24 \mathrm{~h}$ after inhalation $(p \leq 0.05)$. The relative percentage of GP-Av-bEGF fluorescent signals attained a maximum value of $368.3 \pm 15.67 \%$ in the cancerous lungs at the time point of $24 \mathrm{~h}$, while the fluorescence intensity was only $103.87 \pm 39 \%$ in the normal lungs (Fig. 4(b)). Moreover, the fluorescence intensity of the GP-Av-bEGF in the lungs increased by 3.6-fold from the normal lungs to the cancerous lungs, indicating that the GP-Av-bEGF was specific to the tumor tissue. Although highly fluorescent intensity was also observed in the heart, there's no difference between the normal and tumor induced groups. Accumulation of GP-Av-bEGF in the heart was no variant with the time interval after inhalation. The relative fluorescence intensity observed in the kidneys, as shown in Fig. 4(b), suggested that the degree of particle accumulation was lower for the tumor-induced group than that for the normal group. The uptake of nanoparticles in the spleen and liver is known to be mediated by the function of the reticuloendothelial system (RES) [3], and our results revealed that the GP-Av-bEGF was effective in preventing splenic uptake at all time points, so as the lower fluorescent intensity in the liver.

At $24 \mathrm{~h}$ after the delivery of the GP-Av-bEGF aerosol, the distribution of nanoparticles in normal mice was in the order of kidneys $>$ heart $>$ liver $>$ spleen $>$ brain $>$ lungs. The accumulation of
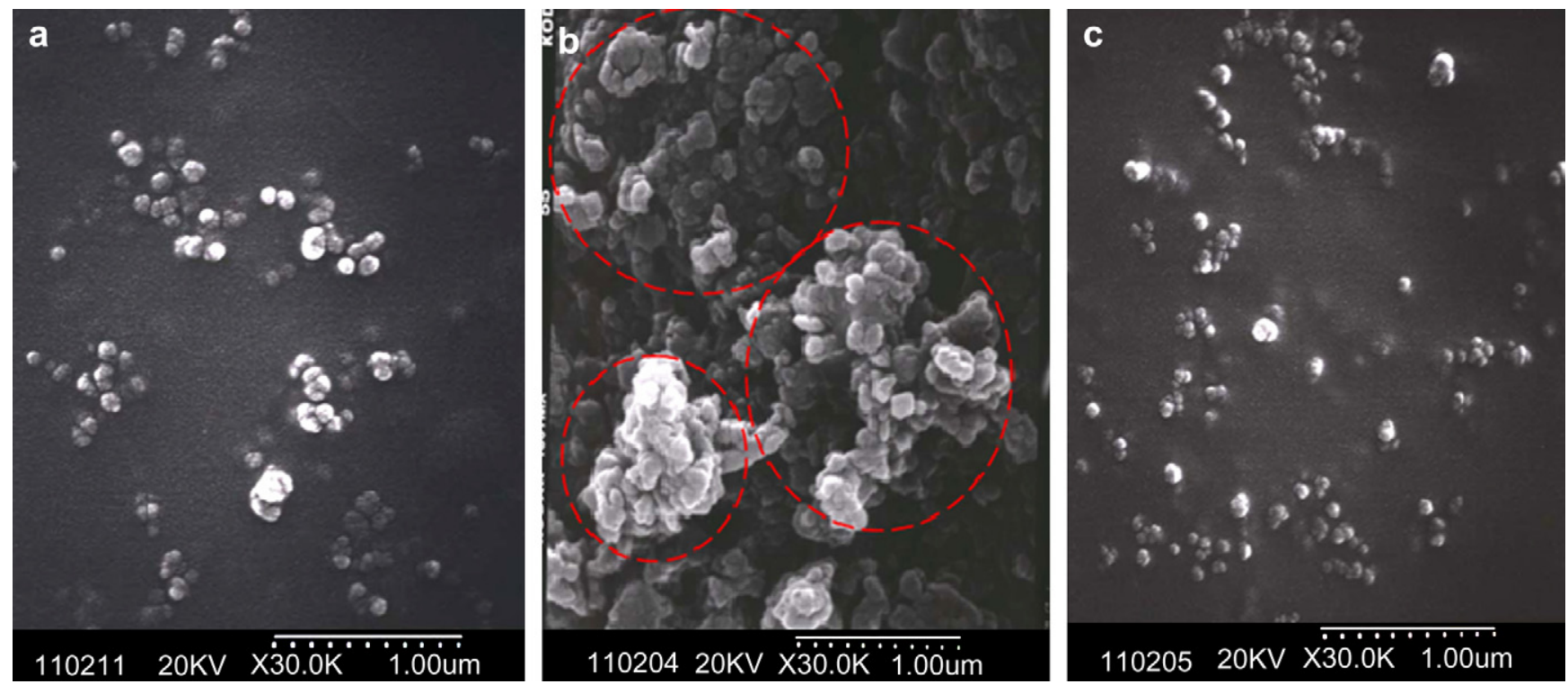

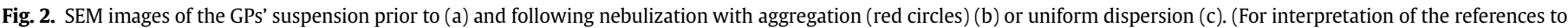
colour in this figure legend, the reader is referred to the web version of this article.) 
a

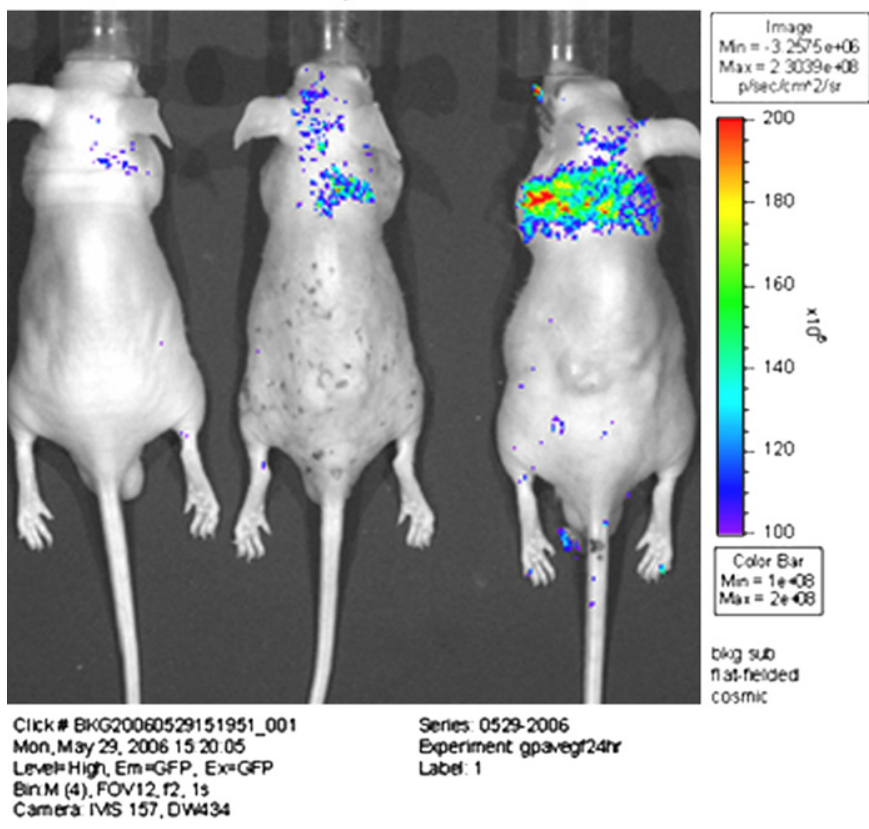

Fig. 3. The in vivo fluorescence images of tumor-induced mice following aerosol delivery $24 \mathrm{~h}$ later by treatment with different nanoparticles' solution: PBS-treated group (a), GP-Av-treated group (b), and GP-Av-bEGF conjugate-treated group (c). FITC green fluorescence spectra were obtained from live mice xenografted with the human lung adenocarcinoma cells (A549).

fluorescent signals was lowest for the lungs and highest for the kidneys. In contrast, GP-Av-bEGF accumulation in the tumorinduced group $24 \mathrm{~h}$ after inhalation was in the order of lungs $>$ heart $>$ kidneys $>$ spleen $>$ brain $>$ liver. In the tumorinduced group, the accumulation in the lungs was significantly greater than that in all the other organs $(p \leq 0.05)$. Further, when compared with the normal group at the same time point, the GPAv-bEGF was gradually eliminated from the spleen and liver, and their accumulation increased in the lungs and kidneys. In particular, the fluorescence intensity for GP-Av-bEGF in the cancerous lungs was considerably higher than that in all the normal tissues examined $(p \leq 0.05)$.

\subsection{Histological examination of the nanoparticle distribution}

Lung sections were examined for FITC expression by performing fluorescence microscopy. By performing hematoxylin-eosin (H\&E) staining (Fig. 5(a)), small spherical particles were observed in the respiratory tract; these may have been GPs stained with eosin. Compared with fluorescence image, aerosol administration of fluorescently labeled GP-Av-bEGF revealed the nanoparticles to be mainly localized to the epithelial cells lining the trachea and the bronchioles of the mid-airways $0.5 \mathrm{~h}$ after the administration (Fig. 5(b)). The nanoparticles were associated with the trachea above the epithelial cell layer. Green fluorescent spots were observed in lines along the respiration tract; however, no fluorescent signals were observed in the blood vessel due to the presence of red blood cells initially (Fig. 5(c)). For sections of $24 \mathrm{~h}$ after the administration, green fluorescent spots were found in the blood vessel (data not shown). Analysis of the lung sections revealed that the GP-Av-bEGFs were principally expressed by the epithelial cells (Fig. 5(d)).

To further confirm the localization of the EGF probe within the tumor tissue, cryosections were obtained from tumor samples $24 \mathrm{~h}$ after the delivery of the GP-Av-EGF aerosol and were processed by H\&E staining and immunohistological staining with anti-EGFR. As shown in Fig. 6(a), a small tumor nodule was observed by H\&E staining, accompanied by significant fluorescence signals from the dye-conjugated GP-Av-bEGF (Fig. 6(b)). On comparing the sections stained with dye-conjugated GP-Av-bEGF and those stained with anti-EGFR, dark brown pigmentation reflecting EGFRoverexpressing cells was observed in the same regions. The fluorescent images revealed that the GP-Av-bEGF signals corresponded to EGFR-expressing cells. Based on these results, we confirmed that the GP-Av-bEGF targeted the EGFR-overexpressing cancer cells.

\section{Discussion}

The treatment of lung cancer continues to be a challenge for oncologists. The topical delivery of anticancer agents to the lungs via aerosols offers the advantage of achieving higher pulmonary concentrations of the therapeutic agent by using a lower total dose than that required for systemic administration, thus lowering the risk of toxicity [16]. Therefore, the direct delivery of chemotherapeutic agents to the lungs could represent a novel therapeutic approach for patients with pulmonary metastases [17]. Inhalation treatment is a noninvasive modality that can be performed at home under supervision, thus reducing the frequency of clinical visits and yielding greater freedom to patients. The present study was designed to expand our understanding of GPs modified with EGF ligand (GP-Av-bEGF) after aerosol delivery could be transferred to the lungs and trace to lung cancer cells in vivo for future clinical applications.

The mucociliary clearance (MCC) is an integral part of the defense mechanism of the lungs and enables the efficient clearance of inhaled drugs, particles, or microorganisms from the respiratory tract [18]. Many studies have focused on the size of the primary particles; however, the size of the aerosol droplet determines whether the particles reach the target site. Particles are deposited in the upper respiratory tract when the aerosol particle size is greater than $10 \mu \mathrm{m}$. If an aerosol is required to be localized at the bronchi or bronchial tubes, it should be between 1 and $5 \mu \mathrm{m}$. Particles in a size of $0.5-1 \mu \mathrm{m}$ are delivered to the pulmonary alveoli, while particles smaller than $0.5 \mu \mathrm{m}$ are exhaled [19]. The deposition of particles in the lungs depends on its mass median aerodynamic diameter (MMAD). Particles having a narrow size distribution and an MMAD in the range $1-5 \mu \mathrm{m}$ are desirable for highly efficient delivery of drugs to the lungs via inhalation [20]. The direct pulmonary delivery of nanoparticles is difficult because they are immediately exhaled during the next breath and only an extremely small percentage of the nanoparticles were actually deposited in the deeper regions of the lungs. The GPs with EGF modification were approximately $220 \mathrm{~nm}$ [15] and thus could have been immediately exhaled on direct delivery to the lungs. Therefore, a device capable of generating and delivering droplets/particles having the requisite MMAD is required.

A nebulizer is known to generate droplets having characteristics suitable for lung deposition [21]. In this study, a nebulizer was used to produce aerosol particles ranging from 0.5 to $5 \mu \mathrm{m}$. As shown in Fig. 1(b), by using a nebulizer, microdroplets of GPs, GP-Av, and GPAv-bEGF suspension were generated (99.8\%) in the ideal MMAD range and could be delivered and deposited beyond the increasingly narrow airways into the alveoli.

Studies performed using inhaled nanoparticles dispersed in aqueous droplets suggest that MCC can be overcome by nanoparticle [22]. Therefore, nanoparticles are potential vehicles for the efficient transport of drugs to the epithelium, without unwanted MCC. Nanoparticles encapsulated in an aerosol droplet were socalled "Trojan" particles [23]. Trojan particles are prepared by the assembly of nanoparticles derived from different materials into microparticles. On delivery into the peripheral airways, these microparticles liberate their nanoparticle components [2]. Nanoparticles are able to escape clearance mechanisms and provide 

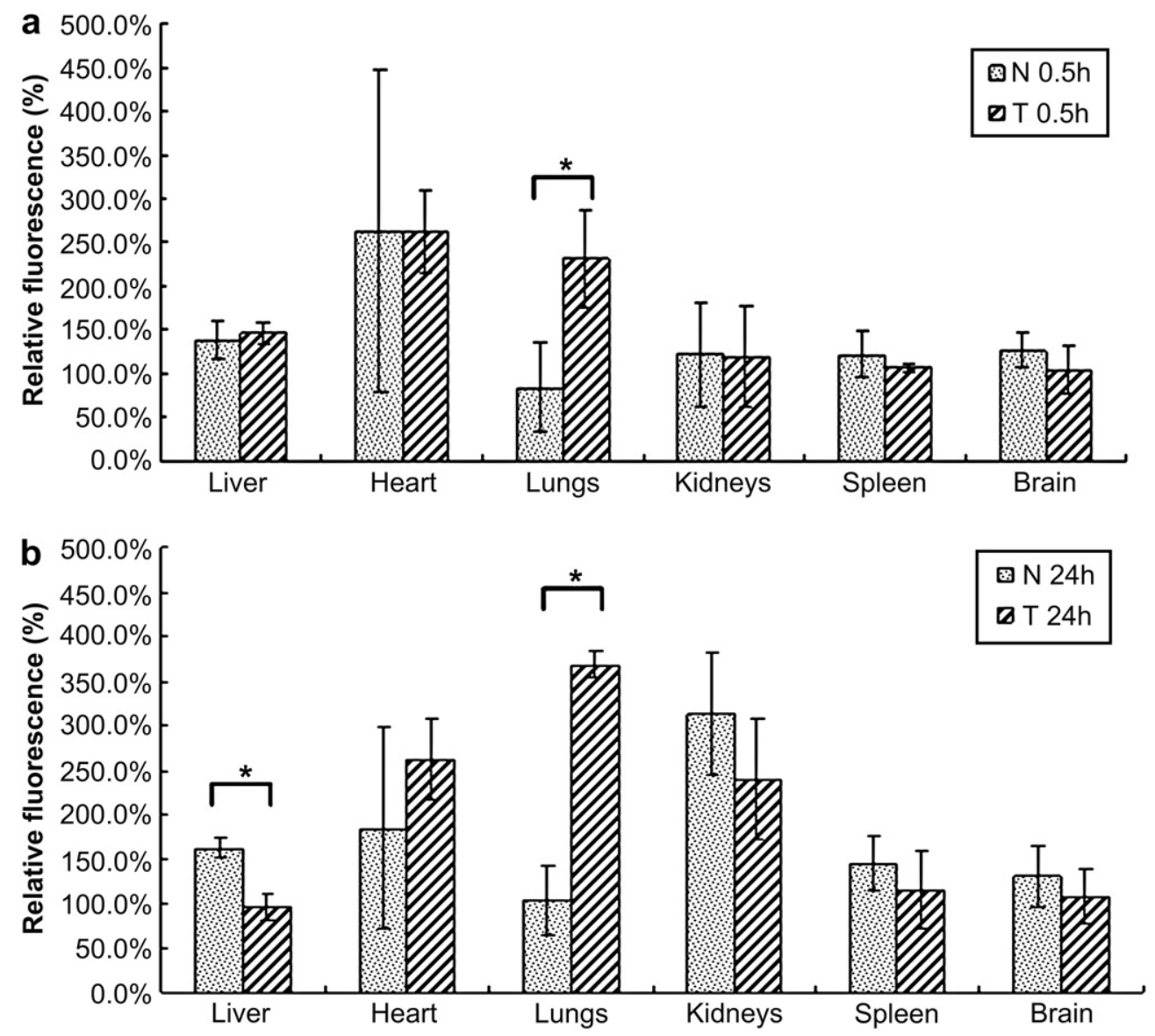

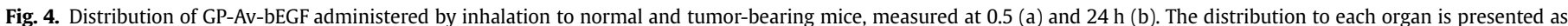

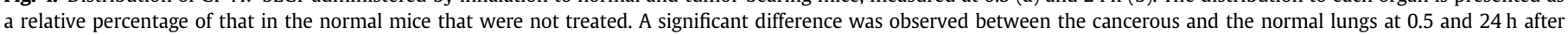
inhalation. The level of significance was considered as $p \leq 0.05\left(^{*}\right)$.

sustained drug release. Our results revealed the formation of Trojan particles (Fig. 2(b)); microparticles were assembled by the aggregation of nanoparticles. We also observed that these nanoparticles could be liberated from the microparticles and redispersed as a single nanoparticle (Fig. 2(c)).

Nanoparticles exhibit a larger surface area per unit mass than larger particles, thus eliciting a greater inflammatory response in the lungs $[24,25]$. Although gelatin has been used extensively in pharmaceuticals, the safety of GPs delivered via inhalation is unknown; therefore lung inflammation examinations were performed. The lung edema was assessed based on the lung $W / D$ weight ratio. The values showed in Table 1 were lower than 6.5. These data were consistent with the previous assessment of acute lung injury study. Normal mice have a $W / D$ weight ratio in the range of 4-6.5, while the ratio in severe lung edema increased significantly with a value higher than 6.5 [26]. From this assay, it appears the GP-Av-bEGF was not responsible for the development of pulmonary edema. Increased MPO activity and increased capillary permeability play an important role in the development of pulmonary injury. In the normal nude mice, no obvious difference in the MPO activity was noted on treatment with PBS and the GPAv-bEGF. This implies that the GP-Av-bEGF did not cause lung inflammation. The MPO activity was lower in the tumor-induced mice treated by the GP-Av-bEGF (Table 1). MPO activity is not only an indication for lung inflammation but has also been shown to be involved in numerous diseases such as atherosclerosis and lung cancer [27]. The reduction in MPO activity observed in the tumorinduced mice treated with the GP-Av-bEGF may have been caused by the induction of tumor and not by lung inflammation. Based on these data, we confirmed that the lungs were not in an inflammation state following nanoparticle inhalation. Therefore, the use of aerosols is a safe method for GP-Av-bEGF delivery. The physicochemical properties of the particulate surface also play an important role in its biological effects on the lungs and in systemic circulation [28]. Unmodified and negatively charged ultrafine particles exert no effect on thrombus formation, whereas positively charged nanoparticles enhance thrombus formation when administrated i.v. [29]. The zeta potential of the GP-Av-bEGF was negative [15], suggesting that these particles may have no risk of thrombus.

Specific uptake depended on EGFR-mediated endocytosis of the drug carrier with EGF or anti-EGFR modification has been developed [30-32]. The internalization of the EGF is reported to occur faster than that of the anti-EGFR antibody in EGFR-overexpressing cells [33]. Therefore, EGF is more favorable than the anti-EGFR for conjugation with nanoparticles for in vivo application. As shown in Fig. 3, among the cancerous mice, the fluorescence intensity was highest in the mouse treated with the GP-Av-bEGF, intermediate in the mouse treated with the GP-Av, and almost absent in that treated with PBS. Sznajder et al. [34] demonstrated that the in vivo delivery of EGF aerosols upregulates the alveolar epithelial ATPase in rats and that EGF retains its biological activity following aerosolization. The data performed in Fig. 3 reveal that GP-Av-bEGF signals could still be traced to the cancer cells indicating that EGF was functional even after aerosolization by using a nebulizer. Under in vivo conditions, EGF probes can be delivered to the tumor site by both passive and active targeting mechanisms. In the case of passive targeting, nanoparticles preferentially accumulate at tumor 


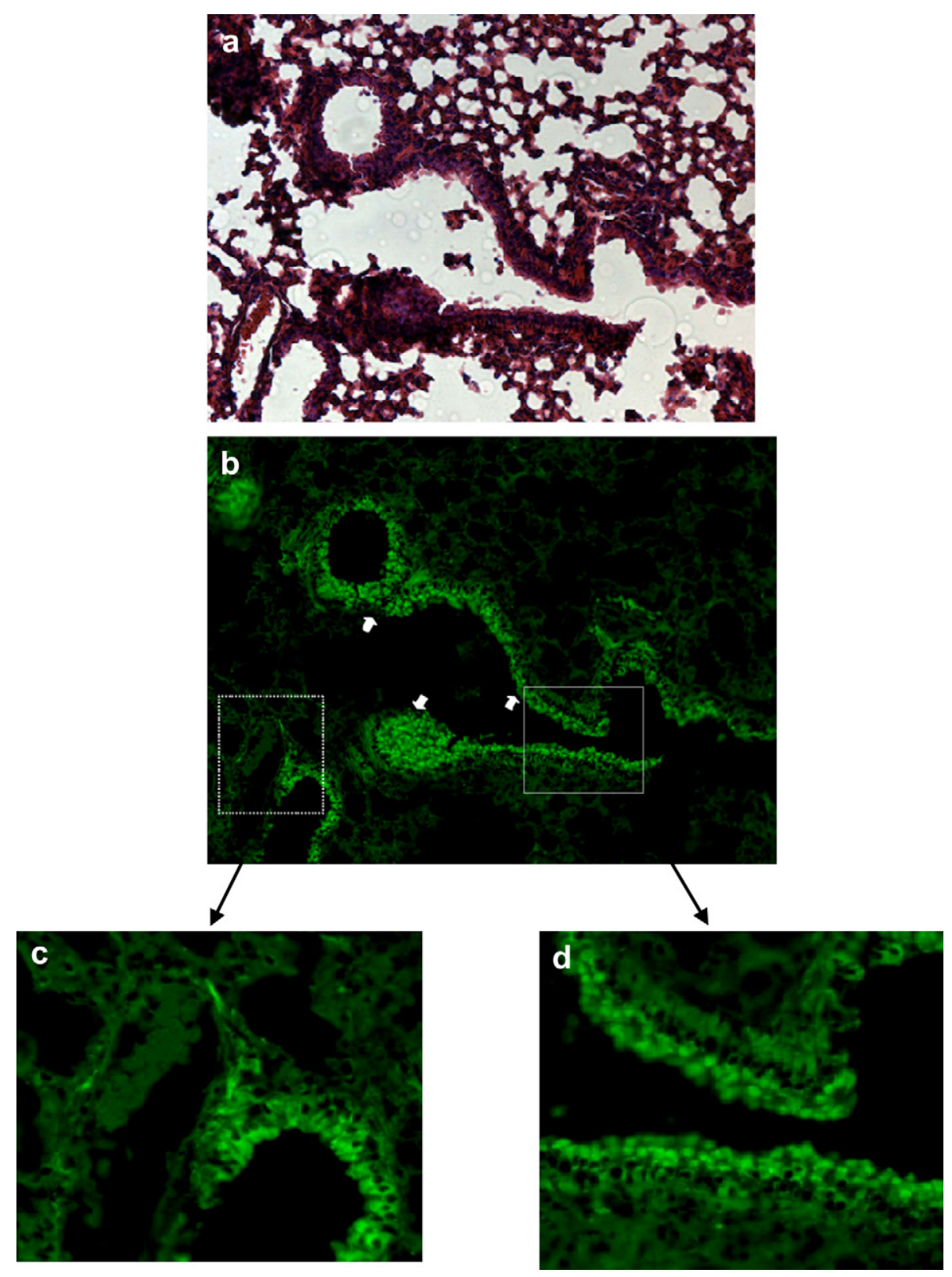

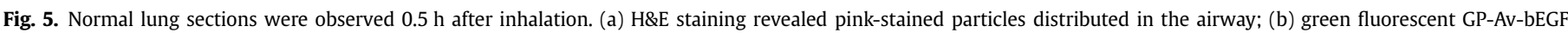

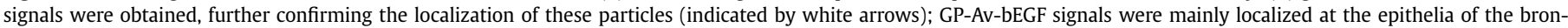

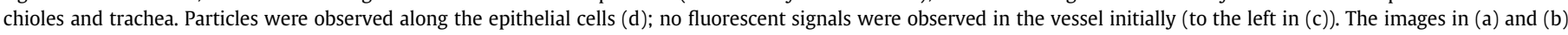
were acquired using a constant microscope setting; magnification, 100×. The images in (c) and (d) are enlarged images of those in (b).

sites via an enhanced permeability and retention (EPR) effect [35]. The GP-Av-bEGF was able to target lung cancer cells in vivo; otherwise, the GP-Av could not recognize tumor cells in cancerous lung. According to this result, we believed that the in vivo targeting ability of the GP-Av-bEGF was contributed to the active targeting mechanism via EGF-EGFR binding.

In the gas exchanged area, the barrier between the alveolar wall and the capillaries is very thin and is separated from the blood flow by only approximately $0.5 \mu \mathrm{m}$ [36] and nanoparticles can be efficiently exchanged from the epithelium to the circulation. In the present study, the GP-Av-bEGF entered the vascular system and was transferred to the whole body, since the nanoparticles were observed in the heart, liver, and kidneys and occasionally in the spleen and brain (Fig. 4). And the lung accumulation of GP-Av-bEGF in tumor group is much higher than that in normal group at 0.5 and $24 \mathrm{~h}$ after inhalation. The accumulation of particles in the lung cancer group $24 \mathrm{~h}$ after inhalation was higher than that $0.5 \mathrm{~h}$ after inhalation. According to this, we hypothesized that some fraction of the GP-Av-bEGF was not trapped by the epithelial cells immediately after inhalation but entered into blood circulation; once these particles approached the lung region again, they could be recognized and taken up by cancer cells with EGFR-overexpression. This tendency was only observed in the lung cancer group, while in the normal mice, there was no difference in the results obtained at $0.5 \mathrm{~h}$ and those obtained at $24 \mathrm{~h}$. This observation reveals that the high accumulation in the lungs of GP-Av-bEGF should depend on EGF ligand guiding, not the simple effect of local delivery via inhalation.

Previous study about the distribution of polyethylene glycol (PEG)-modified gelatin nanoparticles following i.v. administration [3] demonstrated that the relatively low uptake of GPs by the liver was considered to be due to the long-term circulating property 

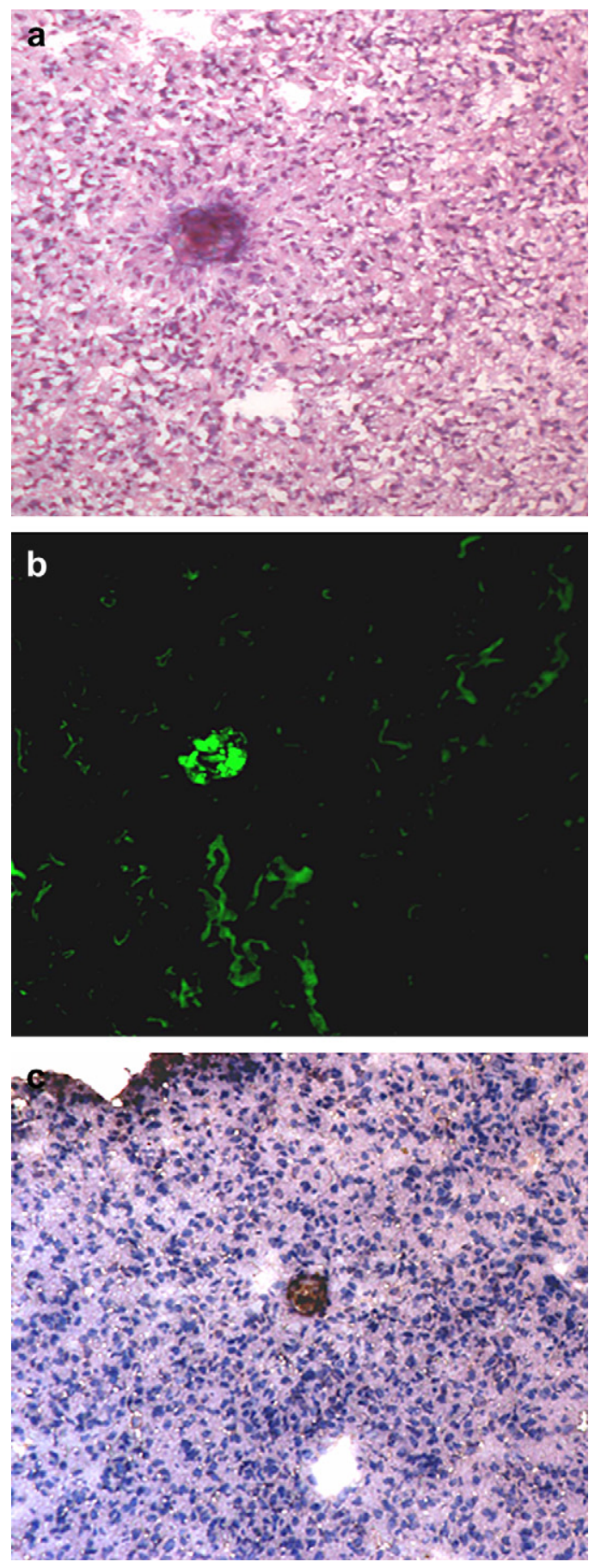

Fig. 6. Histopathological findings of the cancerous lung sections $24 \mathrm{~h}$ after inhalation of the vehicle. (a) H\&E staining revealed the formation of a small nodule; (b) fluorescence imaging confirmed the localization of the particles; (c) immunohistological staining with anti-EGFR revealed dark brown pigmentation, reflecting EGFR-overexpressing cells, in the same region. Magnification, $200 \times$.

imparted to the nanoparticles by surface-accessible PEG chains. PEG could prevent complement activation and macrophageinduced opsonization by the RES following systemic administration [37]. In our study, data revealed that the particle accumulation was lower in the RES, and most of the GP-Av-bEGF was initially caught by EGFR-overexpressing cells in the cancerous lungs; the other particles distributed in blood circulation mainly accumulated in the kidney and heart, followed by the liver and spleen in tumorinduced mice. Fluorescence analysis of the organs showed GP-AvbEGF was lower deposition in spleen and liver; it revealed that
GP-Av-bEGF could escape from the RES system. Due to this result, GP-Av-bEGF nanoparticles, without PEG modification, could match up to the PEG-modified gelatin nanoparticles. Compared with the normal mice, fewer GP-Av-bEGF was observed in the kidneys in cancer-induced mice because of the specific accumulation in the lungs, and therefore we believe that GP-Av-bEGF may serve as a good carrier for the delivery of nephrotoxic drugs to prevent the side effect of kidney damage. Three days after the inhalation, no fluorescence was detected (data not shown), indicating that the particles were completely eliminated from the mice after the treatment was discontinued.

According to these results, the delivery of gelatin nanoparticles as an aerosol in vivo is speculated as below: the nanoparticle solution was nebulized into microdroplet of a suitable MMAD that could escape removal by MCC for aerosol delivery to the lower respiratory track. The aerosol droplets were transported to the alveoli, and the nanoparticles were liberated from the aerosol droplets when in contact with the alveolar wall. Further, the nanoparticles could cross the alveolar barrier and enter the blood vessels; they were subsequently transported in blood circulation to the whole body. Gelatin nanoparticles modified with EGF (GP-AvbEGF) could be recognized and trapped by EGFR-overexpression cancer cells, resulting in a greater degree of accumulation in the cancerous lung tissue.

\section{Conclusion}

The GP-Av-bEGF efficiently bound to the EGFR-overexpressing cells in the mice model of lung cancer. We determined that aerosol droplets of a suitable MMAD formed using these nanoparticles were deposited at the lower respiratory tract, and we demonstrated that jet nebulization does not alter the targeting functions of EGF. In the mice model, we observed the GP-Av-bEGF mainly accumulated in the cancerous lung tissue by using a live imaging procedure for monitoring aerosol deposition. Histological examination revealed that the GP-Av-bEGF was selectively taken up via the EGF receptor on the cancer cells due to the overlapping of fluorescent particles and immunostaining of anti-EGFR. We also demonstrated that the GPs delivered by inhalation do not cause lung inflammation and are thus safe for use. Based on these preclinical results, this noninvasive and convenient system involving the aerosol delivery of nanoparticle drug carriers may be used as an alternative treatment modality for lung cancer in the future.

\section{References}

[1] Koshkina NV, Waldrep JC, Roberts LE, Golunski E, Melton S, Knight V. Paclitaxel liposome aerosol treatment induces inhibition of pulmonary metastases in murine renal carcinoma model. Clin Cancer Res 2001;7:3258-62.

[2] Cryan SA. Carrier-based strategies for targeting protein and peptide drugs to the lungs. AAPS J 2005;7(1):E20-40.

[3] Kaul G, Amiji M. Biodistribution and targeting potential of poly(ethylene glycol) modified gelatin nanoparticles in subcutaneous murine tumor model. J Drug Target 2004;12:585-91.

[4] Kim HW, Park IK, Cho CS, Lee KH, George R, Beck J, et al. Aerosol delivery of glucosylated polyethylenimine/phosphatase and tensin homologue deleted on chromosome 10 complex suppresses Akt downstream pathways in the lung of K-ras null mice. Cancer Res 2004:64:7971-6.

[5] Suarez S, Gonzalez-Rothi RJ, Schreier H, Hochhaus G. Effect of dose and release rate on pulmonary targeting of liposomal triamcinolone acetonide phosphate. Pharm Res 1998;15:461-5.

[6] Deaton AT, Jones LD, Dunbar CA, Hickey AJ, Williams DM. Generation of gelatin aerosol particles from nebulized solutions as model drug carrier systems. Pharm Dev Technol 2002; 7:147-53.

[7] Dailey LA, Jekel N, Fink L, Gessler T, Schmehl T, Wittmar M, et al. Investigation of the proinflammatory potential of biodegradable nanoparticle drug delivery systems in the lung. Toxicol Appl Pharmacol 2006;215:100-8.

[8] Shek PN, Suntres ZE, Brooks JI. Liposomes in pulmonary applications: physicochemical considerations, pulmonary distribution and antioxidant delivery. J Drug Target 1994;2:431-42. 
[9] Peppas NA. An introduction to materials in medicine. In: Ratner BD, Hoffman AS, Schoen FJ, Lemons JE, editors. Biomaterials science. New York: Academic Press; 1996. p. 60-4.

[10] Morimoto K, Katsumata H, Yabuta T, Iwanaga K, Kakemi M, Tabata Y, et al. Gelatin microspheres as a pulmonary delivery system: evaluation of salmon calcitonin absorption. J Pharm Pharmacol 2000;52:611-7.

[11] Farivar AS, MacKinnon-Patterson B, Barnes AD, Mulligan MS. The effect of antiinflammatory properties of mycophenolate mofetil on the development of lung reperfusion injury. J Heart Lung Transplant 2005;24:2235-42.

[12] Coester CJ, Langer K, Briesen HV, Kreuter J. Gelatin nanoparticles by two step desolvation - a new preparation method, surface modifications and cell uptake. J Microencapsul 2000;17:187-93.

[13] Weber C, Reiss S, Langer K. Preparation of surface modified protein nanoparticles by introduction of sulfhydryl groups. Int J Pharm 2000;211: 67-78.

[14] Hermanson GT. Antibody modification and conjugation: bioconjugate techniques. San Diego: Academic Press; 1996.

[15] Tseng C-L, Wang T-W, Dong G-C, Wu SY-H, Young T-H, Shieh M-J, et al. Development of gelatin nanoparticles with biotinylated EGF conjugation for lung cancer targeting. Biomaterials 2007;28:3996-4005.

[16] Koshkina NV, Agoulnik IY, Melton SL, Densmore CL, Knight V. Biodistribution and pharmacokinetics of aerosol and intravenously administered DNApolyethyleneimine complexes: optimization of pulmonary delivery and retention. Mol Ther 2003;8:249-54.

[17] Koshkina NV, Kleinerman ES, Waldrep C, Jia S-F, Worth LL, Gilbert BE, et al. 9-Nitrocamptothecin liposome aerosol treatment of melanoma and osteosarcoma lung metastases in mice. Clin Cancer Res 2000;6:2876-80.

[18] Knowles M, Boucher R. Mucus clearance as a primary innate defense mechanism for mammalian airways. J Clin Invest 2002;109(5):571-7.

[19] Guyton AC, Hall JE. Textbook of medical physiology. 9th ed. Singapore: Harcour Asia Pte Ltd; 2000.

[20] Zanen P, Lammers JW. Reducing adverse effect of inhaled fenoterol through optimization of the aerosol formulation. J Aerosol Med 1999;12: 241-7.

[21] Dalby R, Tiano S, Hickey AJ. Medical devices for the delivery of therapeutic aerosols to the lungs. In: Inhalation aerosols: physical and biological basis for therapy. New York: Marcel Dekker, Inc; 1996.

[22] Schurch S, Gehr P, Im Hof V, Geiser M, Green F. Surfactant displaces particles toward the epithelium in airways and alveoli. Respir Physiol 1990;80: $17-32$.
[23] Tsapis N, Bennett D, Jackson B, Weitz DA, Edwards DA. Trojan particles: large porous carriers of nanoparticles for drug delivery. Proc Natl Acad Sci USA 2002;99:12001-5.

[24] Nemmar A, Vanbilloen H, Hoylaerts MF, Hoet PHM, Verbruggen A, Nemery B. Passage of intratracheally instilled ultrafine particles from the lung into the systemic circulation in hamster. Am J Respir Crit Care Med 2001;164:1665-8.

[25] MacNee W, Donaldson K. How can ultrafine particles be responsible for increased mortality. Monaldi Arch Chest Dis 2000;55:135-9.

[26] Zhou Z, Kozlowski J, Schuster DP. Physiologic, biochemical, and imaging characterization of acute lung injury in mice. Am J Respir Crit Care Med 2005; 172:344-51.

[27] Hoy A, Muller BL, Kutter D, Siest G, Visvikis S. Growing significance of myeloperoxidase in non-infectious diseases. Clin Chem Lab Med 2002:40:2-8.

[28] Geys J, Coenegrachts L, Vercammen J, Engelborghs Y, Nemmara A, Nemery B et al. In vitro study of the pulmonary translocation of nanoparticles: a preliminary study. Toxicol Lett 2006;160:218-26.

[29] Nemmar A, Hoylaerts MF, Hoet PHM, Dinsdale D, Smith T, Xu H, et al. Ultrafine particles affect experimental thrombosis in an in vivo hamster model. Am J Respir Crit Care Med 2002;166:998-1004.

[30] Nielsen UB, Kirpotin DB, Pickering EM, Hong K, Park JW, Shalaby MR, et al. Therapeutic efficacy of anti-ErbB2 immunoliposomes targeted by a phage antibody selected for cellular endocytosis. Biochim Biophys Acta 2002;1591: 109-18.

[31] Carlsson J, Blomquist E, Gedda L, Liljegren A, Malmstroerm P-U, Stroerm AS, et al. Conjugate chemistry and cellular processing of EGF-dextran. Acta Onco 1999;38:313-21.

[32] Raben D, Helfrich B, Chan DC, Ciardiello F, Zhao L, Franklin W, et al. The effects of cetuximab alone and in combination with radiation and/or chemotherapy in lung cancer. Clin Cancer Res 2005;11:795-805.

[33] Fan Z, Lu Y, Wu X, Mendelsohn J. Antibody-induced epidermal growth factor receptor dimerization mediates inhibition of autocrine proliferation of A431 squamous carcinoma cells. J Biol Chem 1994;269:27595-602.

[34] Sznajder JI, Ridge KM, Yeates DB, Ilekis J, Olivera W. Epidermal growth factor increases lung liquid clearance in rat lungs. J Appl Physiol 1998;85:1004-10.

[35] Nie S, Xing Y, Kim GJ, Simons JW. Nanotechnology applications in cancer. Annu Rev Biomed Eng 2007;9:257-88.

[36] Hoet PH, Hohlfeld IB, Salata OV. Nanoparticles - known and unknown health risks. J Nanobiotechnol 2004;2:1-15.

[37] Otsuka H, Nagasaki Y, Kataoka K. PEGylated nanoparticles for biological and pharmaceutical applications. Adv Drug Deliv Rev 2003;55:403-19. 